AdDendum (March 8, 1963). It was pointed out to me by H. R. Pitt that a different proof of the prime number theorem based on $\log n$ ! and using Wiener's theorem was given by A. E. Ingham, Some Tauberian theorems connected with the prime number theorem, J. London Math. Soc. 22 (1945), 161-180.

\title{
REFERENCES
}

1. Edmund Landau, Handbuch der Lehre von der Verteilung der Primzahlen, Vol. 1, 1909, Chelsea, New York, 1953.

2. Norbert Wiener, The Fourier integral and certain of its applications, Cambridge Univ. Press, Cambridge, 1932.

Massachusetts Institute of Technology

\section{FLAG-TRANSITIVE PLANES OF EVEN ORDER ${ }^{1}$}

R. ROTH

1. Introduction. In a projective plane a configuration consisting of a line and a point incident with that line is called a flag. A collineation group which is transitive on the flags of a projective plane is called flag-transitive (or "acutely transitive"). Such a group is also called sharply flag-transitive (or "flag-regular" or "acutely regular") if in addition, the only collineation leaving a flag fixed is the identity. A projective plane is called flag-transitive or sharply flag-transitive, respectively, if it admits a group of collineations which is flag-transitive or sharply flag-transitive, respectively. D. G. Higman and J. E. McLaughlin proved the following theorem [6, Proposition 10, p. 391].

Theorem. Given a finite projective plane of odd order $n$ with a flagtransitive group $G$ where $n$ is not a square or else $n=m^{2}$ and $m \equiv-1$ (mod 4). Then the plane is Desarguesian and the group $G$ is doubly transitive.

In this paper, this theorem is extended to finite projective planes of many orders $n$. It is shown (Theorem 1 ) that for any integer $n$ such that either $n+1$ or $n^{2}+n+1$ is a prime, a flag-transitive plane of order $n$ must be Desarguesian or sharply flag-transitive. However, as

Received by the editors February 14, 1963.

1 This paper represents part of a Ph.D. dissertation written at the University of California, Berkeley, under the supervision of Professor A. Seidenberg. 
was already shown in [6], if $n$ is the order of a sharply flag-transitive plane, then $n^{2}+n+1$ must be a power of a prime (Theorem 2). Thus a contradiction is reached for many integers $n$ unless the flag-transitive plane is Desarguesian, or, if $n$ is not a power of a prime, nonexistent. In addition it is shown that if $n^{2}+n+1$ is not a power of a prime and $n$ is not a square, then a plane of order $n$ with a flag-transitive group is Desarguesian.

The work in this paper does not require $n$ to be even, but the results give little information for odd orders of $n$ not already given by the theorem of Higman and McLaughlin.

It is known that a doubly transitive finite plane is Desarguesian (Theorem 5 in [7, p. 191]) and conjectured that a transitive plane is Desarguesian. Flag-transitivity is stronger than transitivity but weaker than double transitivity. Thus, short of proving that all transitive planes are Desarguesian, it would be desirable to prove that all flag-transitive planes are Desarguesian. The theorem of Higman and McLaughlin quoted and this paper thus represent partial solutions of this problem.

\section{Main results.}

ThEOREM 1. Given a finite plane of order $n$ where either $n^{2}+n+1$ or $n+1$ is a prime and given $G$ a flag-transitive collineation group for the plane. Then $G$ is either doubly transitive or contains a subgroup which is sharply flag-transitive.

Corollary. A flag-transitive plane of order $n$ satisfying the above conditions is either Desarguesian or sharply flag-transitive.

The corollary is immediate from the theorem, since as was just noted, a doubly transitive projective plane is Desarguesian.

For the proof, a number of theorems on permutation groups will be used. [11] is a useful reference.

Definition. A permutation group is called regular if it is transitive but no element other than the identity fixes any letters.

Proposition 1 (Frobenius' Theorem). Given a permutation group $G$ which is transitive but the only element fixing two letters is the identity. Then the elements which displace all the letters, together with the identity, form a normal regular subgroup $M$ (see [5, p. 292]). We call a nonregular permutation group satisfying the above hypotheses a Frobenius group. If $G_{P}$ is the subgroup fixing one letter $P$ then $G=G_{P} M$.

Proposition 2 (Burnside's Theorem). Every nonsolvable transitive group of prime degree is doubly transitive. 
See [11, Theorem 11.7 , p. 36 (and also p. 85 )]. A proof was given by Schur [8] and is essentially reproduced in $[1$, p. 234].

Proposition 3 (Theorem of Galois). Given a transitive permutation group on $p$ letters where $p$ is prime. $G$ is then solvable if and only if any element fixing two letters is the identity.

For the proof see $[11$, p. 35$]$ (see also $[9, \S 60]$ ). We can now proceed to the proof of Theorem 1 .

Case I. $n^{2}+n+1=p$ is a prime. By Proposition 2 applied to the transitive group $G$ we see that $G$ is either doubly transitive or solvable. If it is doubly transitive, that is one of the desired conclusions. If not, it is solvable, so by Proposition 3, only the identity can fix as many as two points. However, $G$ is not regular (it is flag-transitive so there are nontrivial elements fixing a point) hence is a Frobenius group; i.e., it is a group to which Proposition 1 applies nontrivially. By Proposition 1, $G$ has a normal regular subgroup $M . G=G_{P} M$ where $G_{P}$ is the subgroup of $G$ fixing a point $P$. By flag-transitivity of $G$, $G_{P}$ is transitive on the $n+1$ lines through $P$. We regard $G_{P}$ as a transitive permutation group on the $n+1$ lines through $P$ and note that no collineation in $G_{P}$ fixes two of these lines for it would then fix at least two points and contradict the fact that $G$ is a Frobenius group. (A collineation of a finite plane fixes the same number of points as lines. See $\left[5\right.$, p. 400].) Thus $G_{P}$ is either regular or a Frobenius group (on the $n+1$ lines). If it is a Frobenius group call the normal regular subgroup $H$. If $G_{P}$ is already regular let $H=G_{P}$. Then $H M$ is a subgroup of $G_{P} M=G$ (since $M$ is normal), and $H M$ is sharply flagtransitive. Q.E.D.

Case II. $n+1$ is a prime. Consider the representation of $G_{P}$ as a transitive permutation group on the $n+1$ lines through $P$. We may assume that it is a faithful representation for if not, there is some nontrivial collineation in $G_{P} \subset G$ fixing all the lines through $P$. It is thus a central collineation and $G$ is a transitive group containing a central collineation. By a theorem of Wagner (see [10, Theorem 3, p. 122]) the plane is Desarguesian and $G$ contains the (doubly transitive) little projective group.

Hence applying Proposition 2 to $G_{P}$ represented faithfully as a transitive group on the $n+1$ lines through $P$ we see that $G_{P}$ is either doubly transitive or solvable. If $G_{P}$ is doubly transitive on the $n+1$ lines through $P$, it is easy to show that $G$ (being transitive on the points) is doubly transitive on all the lines. It is well known that $G$ is therefore doubly transitive on the points (see, for example [7, corollary to Lemma 2, p. 193]). 
Suppose instead that $G_{P}$ is solvable. Again, Proposition 3 shows that no element of $G_{P}$ fixes two lines, so $G_{P}$ is either a regular group or a Frobenius group (on the $n+1$ lines). If $G_{P}$ is regular, set $H=G_{P}$. If it is a Frobenius group let $H$ be the regular normal subgroup of $G_{P}$ (applying Proposition 1). Now $G$ itself is a Frobenius group (represented on all the points) since no element can fix two points, for otherwise such an element would fix two lines intersecting in a point $Q$ and $G_{Q}$ would have an element fixing two lines. $G_{Q}$ and $G_{P}$ are isomorphic; however, $G_{P}$ has no element fixing two lines, by the above. So applying Proposition 1 to $G$ we see that $G$ has a normal regular subgroup $M$. Then $H M$ is a sharply flag-transitive subgroup of G. Q.E.D.

The next theorem is included in and weaker than Proposition 4 of $[6$, p. 387]. The proof in that paper uses an important new deep result of Thompson: In a Frobenius group $G$ the normal regular subgroup $M$ is nilpotent. The theorem here is proved without Thompson's result.

THEOREM 2. A sharply flag-transitive group is a Frobenius group and moreover the normal regular subgroup $M$ is elementary abelian of prime power order so that $n^{2}+n+1$ is a power of a prime.

Proof. Clearly, a sharply flag-transitive group is a Frobenius group since it is transitive (but not regular) and any element leaving fixed two points would leave fixed the line joining them, thus would fix an incident point-line pair and be the identity. A theorem of Frobenius (see [4], see also [11, Theorem 11.8, p. 36] for statement but not proof) states that the regular normal subgroup of a primitive Frobenius group is elementary abelian. Since Higman and McLaughlin proved in [6, Proposition 3, p. 386] that any flag-transitive group is primitive as a permutation group on the points we may apply the above theorem. Thus $M$ is elementary abelian, hence has prime power order which is equal to $n^{2}+n+1$, the number of points operated on by the group.

THEOREM 3. If $n^{2}+n+1$ is not a power of a prime then a flagtransitive group is nonsolvable.

Proof. We apply Theorem 11.5 of [11]: If $N$ is a minimal normal subgroup of a primitive group $G$ and $N$ is solvable then $N$ is regular and elementary abelian. Hence the degree of $G$ is a power of a prime. As mentioned in the proof of Theorem 2, a flag-transitive group is primitive as a permutation group on the points. Thus if a flagtransitive group is solvable, then $n^{2}+n+1$ is a power of a prime. Q.E.D. 
For the corollary we need a theorem of W. Feit and J. G. Thompson unpublished at the time of this writing (see [3]). It states that any nonsolvable group is of even order.

CoROLlARY. If $n^{2}+n+1$ is not a power of a prime and $n$ is not a square then a plane of order $n$ with a flag-transitive group is Desarguesian.

Proof. By Theorem 3, $G$ is nonsolvable. By the above, $G$ is thus of even order. Hence it has an involution which must be a central collineation since $n$ is not a square [5, Theorem 20.9.7]. Thus $G$ is a transitive group with a central collineation, hence the plane is Desarguesian [10, Theorem 3].

3. Applications. We can now apply these theorems to show that a flag-transitive plane must be Desarguesian for many orders $n$. One line of reasoning is the following: If $n+1$ is a prime then by the corollary to Theorem 1 (Case II) the plane is Desarguesian or sharply flag-transitive. But if $n^{2}+n+1$ is not a power of a prime, Theorem 2 shows that the plane is not sharply flag-transitive. Of course, then if $\boldsymbol{n}$ itself is not a power of a prime, no such plane exists. For instance, if $n=36$, then $n+1=37$, and $n^{2}+n+1=1333=31 \cdot 43$, so there are no flag-transitive planes of order 36 . The same argument works for $n=10,28,40,52,58,60,72,82,88,96,100$. Moreover a similar argument shows that for $n=16$, a flag-transitive plane must be Desarguesian.

Another way of applying these theorems makes use of a result of Evans and Mann (see [2]) that if $n$ is the order of a cyclic plane and $n \leqq 1600$, then $n$ is a power of a prime. Now if $n^{2}+n+1$ is a prime, the corollary to Theorem 1 (Case I) shows that a flag-transitive plane of order $n$ is sharply flag-transitive (if not Desarguesian) and Theorem 2 says that it must possess an elementary abelian regular collineation group of prime order $n^{2}+n+1$ which is therefore cyclic. Hence if $n$ is not a power of a prime, no flag-transitive plane of order $n$ exists (here $n \leqq 1600)$. For example, if $n=20, n^{2}+n+1=421$, a prime, so no flag-transitive plane exists for $n=20$. Similarly no flag-transitive plane exists for $n=12,24,50,80,90$. For the case $n=18$, we have $n+1=19$, so a flag-transitive plane of order 18 would be sharply flag-transitive, which is a contradiction as pointed out in [6, p. 393]. Hence there are no flag-transitive planes of order $n=18$.

The corollary to Theorem 3 shows that for many other integers a flag-transitive plane must be Desarguesian (for example, $n=26$, $\left.n^{2}+n+1=703=19 \cdot 37\right)$. These theorems may thus be applied to show that for all even $n \leqq 100$, with the possible exception of $n=64$, 
a flag-transitive plane of order $n$ must be Desarguesian. Values of $n$ for which the Bruck-Ryser theorem [5, Theorem 20.8.3, p. 394] shows that no projective planes of order $n$ exist are, of course, excluded.

\section{BIBLIOGRAPHY}

1. R. Carmichael, Groups of finite order, Dover, New York, 1956.

2. T. A. Evans and H. B. Mann, On simple difference sets, Sankhyā 11 (1951), 357-364.

3. W. Feit and J. G. Thompson, Solvability of groups of odd order, Pacific J. Math. 13 (1963).

4. G. Frobenius, Über primitive Gruppen des Grades $n$ und der Klasse n-1, S.-B. Deutsch. Akad. Wiss. Berlin Kl. Math. Phys. Tech. (1902), 455-459.

5. M. Hall, Jr., The theory of groups, Macmillan, New York, 1959.

6. D. G. Higman and J. E. McLaughlin, Geometric ABA-groups, Illinois J. Math. 5 (1961), 382-395.

7. T. G. Ostrom and A. Wagner, On projective and affine planes with transitive collineation groups, Math. Z. 71 (1959), 186-199.

8. I. Schur, Neuer Beweis eines Satzes von W. Burnside, Jber. Deutsch. Math.Verein. 17 (1908), 171-176.

9. B. L. van der Waerden, Modern algebra, rev. English ed., Ungar, New York, 1953.

10. A. Wagner, On perspectivities of finite projective planes, Math. Z. 71 (1959), 113-123.

11. H. Wielandt, Permutation groups, Lectures at the University of Tubingen 1954-1955. Notes from California Institute of Technology, Pasadena, Calif.

University of California, Berkeley 